\title{
Common airborne fungi induce species-specific effects on upper airway inflammatory and remodelling responses*
}

\author{
Eleanor L. Sproson ${ }^{1,2 *}$, Kelly M. Thomas ${ }^{1 *}$, Laurie C. Lau', Philip G. Harries², \\ Peter H. Howarth ${ }^{1,3,4}$, Rami J. Salib 1,2,3,4 \\ Academic Unit of Clinical \& Experimental Sciences, Faculty of Medicine, University of Southampton, Southampton, \\ United Kingdom \\ ${ }^{2}$ Department of Otorhinolaryngology - Head and Neck Surgery, University Hospital Southampton NHS Foundation Trust, \\ Southampton, United Kingdom \\ ${ }^{3}$ Southampton NIHR Respiratory Biomedical Research Unit, University of Southampton \& University Hospital Southampton \\ NHS Foundation Trust, Southampton, United Kingdom \\ ${ }^{4}$ Southampton NIHR Wellcome Trust Clinical Research Facility, Southampton, United Kingdom
}

Rhinology 54: 51-55, 2016

DOI:10.4193/Rhino14.278
*Received for publication:
November 13, 2014
Accepted: July 15, 2015
* These authors contributed
equally

\begin{abstract}
Objective: Whilst the exact cause of chronic rhinosinusitis (CRS) remains elusive, it is clear that both inflammation and remodelling are key disease processes. Environmental fungi have been linked to airway inflammation in CRS; however, their role in the pathogenesis of this condition remains controversial. The current consensus suggests that whilst fungi may not be directly causative, it is likely that CRS patients have deficits in their innate and potentially acquired immunity, which in turn may modify their ability to react to fungi. This study used a nasal polyp explant tissue stimulation model to study the inflammatory and remodelling responses related to challenge with common airborne fungal species.
\end{abstract}

Methods: Ex vivo nasal polyp tissue from six well phenotyped CRSwNP patients undergoing functional endoscopic sinus surgery was stimulated with 1, 10 and $100 \mu \mathrm{g} / \mathrm{ml}$ of Alternaria alternata, Aspergillus niger, Cladosporium sphaerospermum and Penicillium notatum and compared with unchallenged polyp tissue as control. Enzyme-linked immunosorbent assay (ELISA) was used to measure the levels of pro-inflammatory cytokines interleukin-6 (IL-6), granulocyte macrophage colony stimulating factor (GMCSF) and tumour necrosis factor-a (TNF-a); and pro-remodelling cytokines transforming growth factor- $\beta 1$ (TGF- $\beta 1$ ), and basic fibroblast growth factor (bFGF) in the polyp supernatant.

Results: Aspergillus niger stimulation increased pro-inflammatory cytokines TNF-a, GM-CSF and IL-6 whilst having little effect on the remodelling cytokines bFGF and TGF- $\beta 1$. In contrast, stimulation with Cladosporium sphaerospermum, Alternaria alternata and Penicillium notatum reduced pro-inflammatory cytokines TNF- $\alpha$ and IL-6, but induced a dose-dependent increase in remodelling cytokines TGF- $\beta 1$ and bFGF.

Conclusions: This study shows that common airborne fungi induce species-specific effects on the upper airway inflammatory and remodelling responses. These findings provide further immunological evidence of a disease-modifying role for fungi in CRS.

Key words: Chronic rhinosinusitis, nasal polyps, fungus, cytokines, inflammatory, remodelling

\section{Introduction}

Chronic rhinosinusitis (CRS) with or without nasal polyps (CRSwNP/CRSsNP) is one of the commonest conditions encountered in medicine and affects up to $15 \%$ of the general population in Europe and the USA ${ }^{(1)}$. CRS is a disease of the mucosal lining of the sinonasal cavity characterised by recurrent episodes of inflammation resulting in chronic symptoms such as nasal obstruction, facial pain, rhinorrhoea and reduction in sense of smell ${ }^{(1)}$, and has also been closely linked as one of the determinants of asthma severity ${ }^{(2)}$. Whilst the exact cause 
of CRS remains elusive, it is clear that both inflammation and remodelling are key disease processes. Proposed mechanisms of CRS pathophysiology include the role of superantigens, abnormal cell-mediated immune responses, changes in the inflammatory cytokine cascade, epithelial defects, osteitis of the sinus walls and viral, bacterial and fungal factors (1). Despite the link between environmental fungi and $\mathrm{TH} 2$ cell-related airway inflammation in CRS, their role in the pathogenesis of this condition remains controversial. The current consensus suggests that whilst fungi may not be directly causative, it is likely that CRS patients have deficits in their innate and potentially acquired immunity, which in turn may modify their ability to react to fungi ${ }^{(1)}$. This is supported by the finding that peripheral blood mononuclear cells and T-lymphocytes from CRS patients, but not healthy controls, when presented with certain fungal antigens react by producing cytokines interleukin (IL)- 5 and IL-13 which recruit and activate eosinophils ${ }^{(3)}$. Specific lgE to Alternaria has also been found locally in nasal polyp tissue (4). This pilot study developed and validated a nasal polyp explant tissue stimulation model, and utilized it to further characterize the inflammatory and remodelling responses related to challenge with common airborne fungal species.

\section{Materials and methods}

\section{Subjects}

This study was approved by Southampton and South West Hampshire Research Ethics Committee and all patients gave informed consent. Nasal polyps were collected from six CRSwNP patients undergoing endoscopic sinus surgery at University Hospital Southampton NHS Foundation Trust. Patients' age, gender, smoking history, medication and allergic status were recorded.

\section{Nasal explant model}

Nasal polyps were finely chopped into $2 \mathrm{~mm} 3$ fragments and washed several times with Phosphate buffered saline (PBS), before incubation for 16 hours in RPMI-1640 medium supplemented with $1 \%$ penicillin, $1 \%$ streptomycin and $1 \%$ gentamicin at $37^{\circ} \mathrm{C}$ in $5 \% \mathrm{CO}_{2}$. Approximately five fragments of tissue (approximately $20 \mathrm{mg}$ ) were added to $1 \mathrm{ml}$ of medium and stimulated with 1,10 or $100 \mu \mathrm{g} / \mathrm{ml}$ of fungal extract (Greer laboratories, Lenoir, NC, USA) or buffer alone for 24 hours. Tissue was then removed, weighed and supernatants harvested after centrifugation and stored at $-80^{\circ} \mathrm{C}$ until analysis. The fungal extracts utilised were derived from Alternaria alternata, Aspergillus niger, Cladosporium sphaerospermum and Penicillium notatum. The concentrations of fungal extract used were selected on the basis of results from other groups using $50 \mu \mathrm{g} / \mathrm{ml}$.

Due to the lack of a valid 'healthy' control tissue for nasal polyps, unstimulated nasal polyp tissue was used as the control and all cytokine release expressed as fold increase relative to this control.

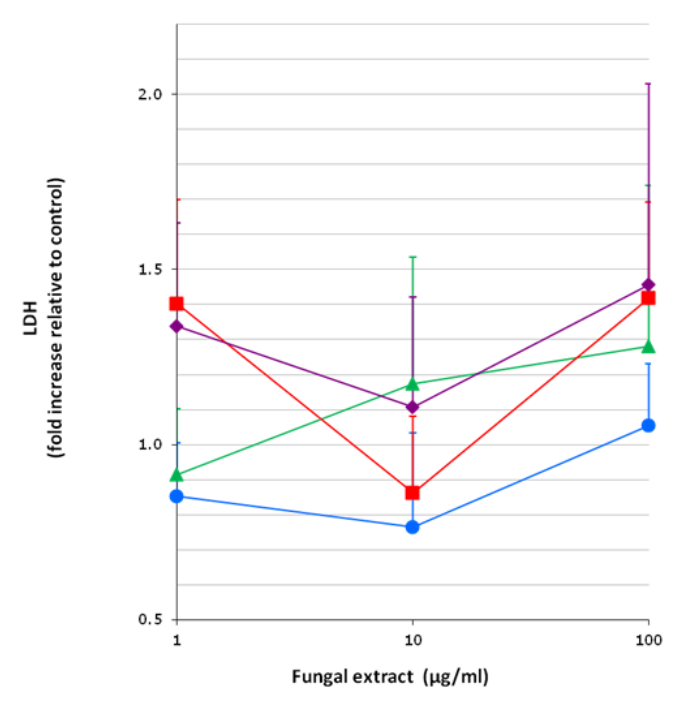

Figure 1. Line graph showing LDH levels following challenge with different concentrations of Aspergillus niger (red), Alternaria alternata (blue), Cladosporium sphaerospermum (green), and Penicillium notatum (purple). Results are expressed as mean \pm SEM of fold increase relative to control (*denotes $\mathrm{p}<0.05)$.

\section{Cytotoxicity of fungal extracts}

A commercially available LDH assay kit (Roche Applied Science, Burgess Hill, UK) was used to assess any cellular cytotoxicity directly related to the fungal extracts. The assay was carried out according to manufacturers' instructions. In summary, the catalyst mixture was reconstituted with $1 \mathrm{ml}$ of double distilled water and mixed for 10 minutes. $250 \mu$ l of this mixture was added to $11.25 \mathrm{ml}$ of the dye solution to form the reaction mixture. $100 \mu$ l of sample or standard was added to a 96-well microtitre plate. $100 \mu$ l of the reaction mixture was added to each well and incubated at room temperature for thirty minutes with continual agitation. The optical density of plates was then read at $490 \mathrm{~nm}$ using a plate reader. LDH concentration was determined by comparing optical density readings with the standard curve.

\section{Enzyme-linked Immunosorbent Assay}

Cytokine levels in the supernatant were determined by enzymelinked immunosorbent assay (ELISA) and corrected for tissue weight. Commercially available ELISA kits for human bFGF, IL-6, GM-CSF, TNF- $\beta$, and TGF- $\beta 1$ (R\&D Systems, Abingdon, UK) were used. Assays were carried out according to manufacturers' instructions. Briefly, 96-well microtitre plates were coated with coating antibody for 16 hours at room temperature. Plates were washed and blocked with $1 \%$ bovine serum albumin in PBS for 2 hours at room temperature. $100 \mu \mathrm{l}$ of sample or standard was added to appropriate wells and plates were incubated at room temperature for two hours with continual agitation. They were then washed four times with $0.05 \%$ PBS-Tween-20 and blotted on paper towel. $100 \mu$ l of anti-bFGF, IL-6, GM-CSF, TNF- $\beta$, or TGF- 

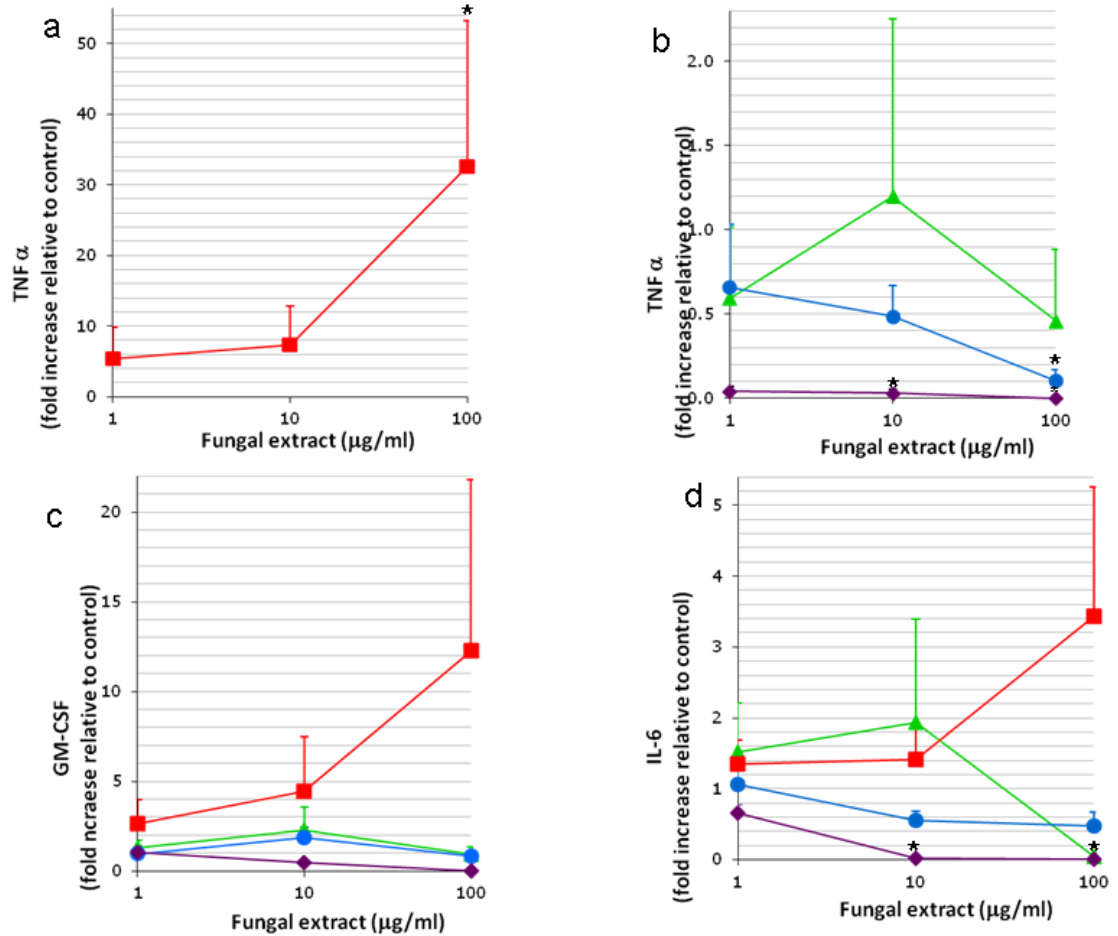

Figure 2. Line graphs showing TNF-a (a \& b), GMCSF (c), IL-6 (d) levels following challenge with different concentrations of Aspergillus niger (red), Alternaria alternata (blue), Cladosporium sphaerospermum (green), and Penicillium notatum (purple). Results are expressed as mean \pm SEM of fold increase relative to control (*denotes $p<0.05)$.

$\beta 1$ detection antibody was added to the plate and incubated for two hours at room temperature with continual agitation. The plate was washed and $100 \mu$ l of streptavidin-horseradish peroxidise (HRP) conjugate added and incubated for 20 minutes. The plates were washed again and $100 \mu \mathrm{l}$ of tetramethylbenzidine (TMB) substrate was added to each well. The reaction between TMB and HRP was terminated using $1 \mathrm{M} \mathrm{H}_{2} \mathrm{SO}_{4}$. The optical density of plates was then read at $450 \mathrm{~nm}$ using a plate reader. Cytokine concentration was determined by comparing optical density readings with the standard curve. The limits of detection were $0.22 \mathrm{pg} / \mathrm{mg}$ tissue for $\mathrm{bFGF}, 0.1 \mathrm{pg} / \mathrm{mg}$ tissue for IL-6, $0.27 \mathrm{pg} / \mathrm{mg}$ tissue for TGF- $\beta 1$ and $0.05 \mathrm{pg} / \mathrm{mg}$ tissue for TNF- $\alpha$.

\section{Statistical analysis}

Results were analysed with InStat ${ }^{\circledast}$ (GraphPad software inc, La Jolla, CA, USA) using non-parametric tests (Friedman and Dunn's multiple comparisons test). A value of $p<0.05$ was considered to be statistically significant.

\section{Results}

\section{Patient demographics}

The samples were collected from 3 males and 3 females with a mean age of $53 \pm 8 \mathrm{yrs}$. Three patients reported allergic-type symptoms and 3 were asthmatic. None were aspirin sensitive. Two patients were taking intranasal corticosteroids at the time of their surgery, but none had taken antibiotics or oral steroids for at least 8 weeks prior to surgery. The patient group contained 2 non-, 2 ex- and 2 current smokers. Skin prick allergy testing to common moulds (indoor \& outdoor) was negative.

Cytotoxicity of fungal extracts

There were no significant differences in LDH levels measured in supernatants from tissue stimulated with any of the fungal extracts compared to unchallenged control tissue (Figure 1).

Pro-inflammatory cytokine release following fungal challenge

TNF- $a$

Aspergillus niger induced a dose-dependent increase in TNF- $a$ release from nasal polyps, with a 30 -fold increase compared to unstimulated control tissue ( $<<0.05$ at $100 \mu \mathrm{g} / \mathrm{ml}$ ). Conversely, Alternaria alternata and Penicillium notatum both appeared to decrease TNF-a release, with the $P$. notatum response reaching significance at $10 \mu \mathrm{g} / \mathrm{ml}$ and A. alternata at $100 \mu \mathrm{g} / \mathrm{ml}$. C. sphaerospermum also displayed the same trend but this did not reach statistical significance (Figures 2A, B).

\section{GM-CSF}

The release of the growth factor GM-CSF again showed a similar pattern of dose-dependent increased release with A. niger and 

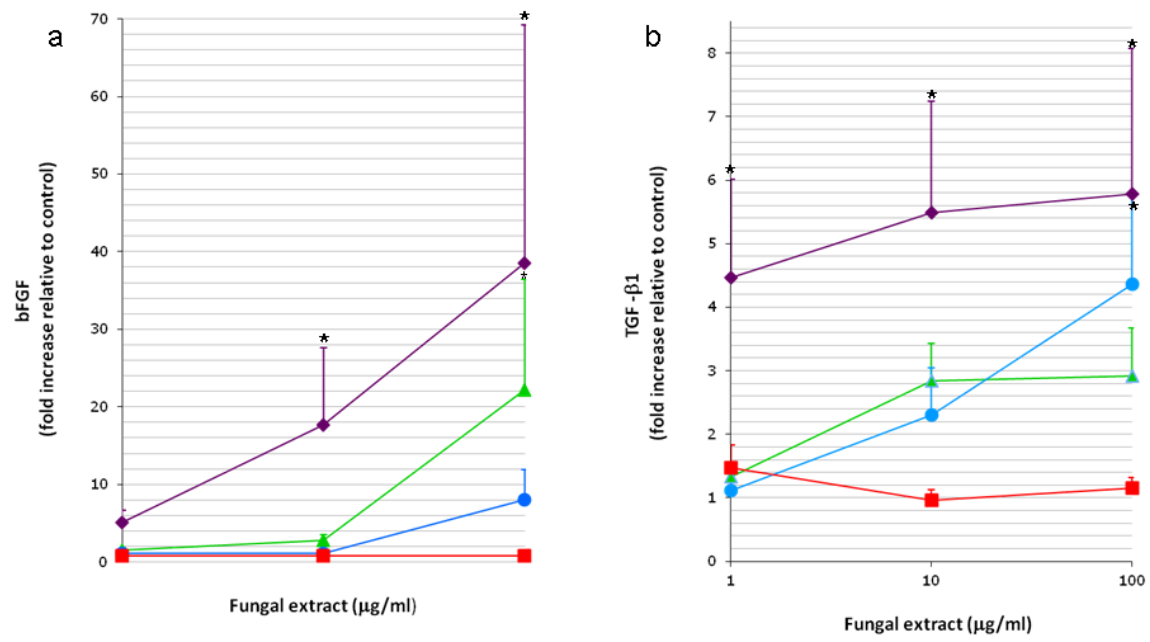

Figure 3. Line graphs showing bFGF (a) TGF- $\beta 1$ (b) levels following challenge with different concentrations of Aspergillus niger (red), Alternaria alternata (blue), Cladosporium sphaerospermum (green), and Penicillium notatum (purple). Results are expressed as mean \pm SEM of fold increase relative to control (*denotes $p<0.05$ ).

decreased release with C. sphaerospermum, A. alternata and $P$. notatum. However, these results did not reach significance (Figure 2C).

\section{IL-6}

IL- 6 showed similar patterns of release in response to the fungal extracts as TNF- $\alpha$. A. niger induced an increase in release of IL-6, although this did not reach statistical significance. In contrast, stimulation with C. sphaerospermum, A. alternata and P. notatum resulted in a decrease in IL-6 levels. In particular, both C. sphaerospermum and $P$. notatum significantly decreased release of IL-6, at 100 and $10 \mu \mathrm{g} / \mathrm{ml}$ respectively (Figure 2D).

Pro-remodelling cytokine release following fungal challenge

bFGF

The remodelling cytokine bFGF shows a distinct pattern of release with C. sphaerospermum, A. alternata and $P$. notatum inducing a dose-dependent increase in bFGF. $P$. notatum induced the greatest response, with an approximately 40 -fold increase after stimulation with $100 \mu \mathrm{g} / \mathrm{ml}(\mathrm{p}<0.05)$, compared with 20 -fold with the same concentration of $C$. sphaerospermum $(p<0.05)$. There was a similar trend for increased release in response to $A$. alternata, but this did not reach statistical significance. Conversely, A. niger does not appear to have any effect on release of bFGF, in contrast to its effect on the release of pro-inflammatory cytokines (Figure 3a).

\section{TGF- $\beta 1$}

The TFG- $\beta 1$ response appeared to mirror that of bFGF. P. notatum significantly increased release of TGF- $\beta 1$ at all 3 concentrations $(p<0.05)$. Similarly A. alternata induced a dose-dependent increase in release of TGF- $\beta 1$, which reached statistical significance at $100 \mu \mathrm{g} / \mathrm{ml}$. C. sphaerospermum also induced dose-dependent release of TGF- $\beta 1$, although this did not reach statistical significance. As with bFGF, A. niger had very little effect on release of TGF- $\beta 1$, with responses fluctuating around the baseline release level in control tissue (Figure $3 b$ ).

\section{Discussion}

With studies showing that they can be cultured from the nasal cavity of most CRS patients ${ }^{(5)}$, fungi have been suggested to play an important role in the pathogenesis of CRS, although this area remains fairly controversial. Furthermore, the results of therapy with topical antifungals including amphotericin have been fairly disappointing with the majority of studies failing to show any significant therapeutic effects ${ }^{(6)}$. Whilst these conflicting results could be a reflection of different dosing regimes and delivery methods, it is now generally accepted that the absence of convincing immunological data or evidence for clinical improvement of CRS with antifungal therapy, suggests that fungi are unlikely to be directly causative agents in the majority of CRS cases ${ }^{(7)}$. Recent studies have shown that there are several deficits in the innate, and potentially also in the acquired immunity of CRS patients ${ }^{(3,4)}$, that might alter their ability to react to fungi. Consequently, and in view of this emerging evidence of intrinsic or induced change in immunity of CRS patients, general opinion now favours fungi as possessing a disease-modifying role in CRS rather than being sole causative agents ${ }^{(1)}$.

This study's results support the concept of fungi as disease modifiers in CRS. In particular, there appears to be a clear speciesspecific distinction in their tissue-related effects, with some primarily inducing pro-inflammatory and others a remodelling cytokine response. Aspergillus niger stimulation increased pro- 
inflammatory cytokines TNF-a, GM-CSF and IL-6 whilst suppressing levels of the main remodelling cytokine TGF- $\beta 1$. In contrast, stimulation with Cladosporium sphaerospermum, Alternaria alternata and Penicillium notatum reduced pro-inflammatory cytokines TNF- $a$, GM-CSF and IL- 6 but induced a dose-dependent increase in remodelling cytokines TGF- $\beta 1$ and bFGF.

It is thought that the effect of fungi on inflammatory and remodelling cytokine production is mediated through interaction between protease-activated receptors (PARs) on nasal epithelial cells and proteases released from the fungi. Several species of fungi have been shown to produce endogenous activators of PARs which can activate epithelial cells to produce chemical mediators ${ }^{(8)}$. In asthma, activation of PARs by fungi has been shown to induce lung inflammation in vivo ${ }^{(9)}$. Protease inhibitors have been shown to block the release of cytokines ${ }^{(8)}$. It is thus feasible that the contrasting effects of fungi on the inflammatory and remodelling cytokine production profiles seen in this study may be protease-dependent, although evidently further work (including PAR expression levels following fungal challenge) will be required in order to substantiate this theory.

\section{Study limitations}

Whilst this is a relatively small study with a degree of heterogeneity and variability, it was designed primarily as a pilot study to test the feasibility of our study design and in particular to develop and validate the use of the human nasal polyp explant tissue stimulation model. Although it is not known whether the concentrations of fungal extracts used reflect those present naturally in the nasal cavity and sinuses, the range of concentrations matched those used in similar studies. Despite these limitations, this study has highlighted some interesting new findings which provide further immunological evidence for a disease-modifying role for fungi in CRS.

\section{Conclusion}

This pilot study characterized the inflammatory and remodelling cytokine responses in ex vivo nasal polyp tissue following challenge with common airborne fungi, and reports contrasting responses which appear to be species-specific. These findings provide further immunological evidence of a disease-modifying role for fungi in CRS.

\section{Authorship contribution}

ES, KT: Laboratory work and manuscript writing; LL: Laboratory work; PHA, PHO: Manuscript revision; RS: Manuscript writing and revision.

\section{Acknowledgement}

This work was supported by a pump priming grant from the Royal College of Surgeons of England (to RJS). PHH and RJS were supported in part by the Southampton National Institute of Health Research (NIHR) Respiratory Biomedical Research Unit and NIHR Wellcome Trust Clinical Research Facility. We are indebted to all the study participants. We would like to thank Dr Jane Warner for her valuable contributions to the development of the tissue stimulation model and for permission to carry out the work in her laboratory. We would also like to thank Mr Scott Harris for his help with the statistical analysis.

\section{Conflict of interest}

None to declare.

\section{References}

1. Fokkens WJ, Lund VJ, Mullol J, et al. EPOS 2012: European position paper on rhinosinusitis and nasal polyps 2012. A summary for otorhinolaryngologists. Rhinology 2012 50:1-12.

2. Bousquet J, Khaltaev N, Cruz AA, et al. Allergic Rhinitis and its Impact on Asthma (ARIA) 2008 update (in collaboration with the World Health Organization, GA(2)LEN and AllerGen). Allergy 2008; 63(Suppl 86):8160.

3. Shin S-H, Ponikau JU, Sherris DA et al Chronic rhinosinusitis: an enhanced immune response to ubiquitous airborne fungi. J. Allergy Clin. Immunol. 2004; 114:1369-75.

4. Sabirov A, Hamilton RG, Jacobs JB, et al. Role of local immunoglobulin E specific for Alternaria alternata in the pathogenesis of nasal polyposis. Laryngoscope 2008; 118:49.

5. Ponikau JU, Sherris DA, Kern EB, et al. The diagnosis and incidence of allergic fungal sinusitis. Mayo Clin. Proc. 1999;74:877-84.
6. Ebbens FA, Georgalas C, Luiten S, et al. The effect of topical amphotericin B on inflammatory markers in patients with chronic rhinosinusitis: a multicenter randomized controlled study. Laryngoscope 2009; 119:401-408.

7. Fokkens WJ, Van Drunen C, Georgalas C, et al. Role of fungi in pathogenesis of chronic rhinosinusitis: The hypothesis rejected. Curr. Opin. Otolaryngol. Head Neck Surg. 2012; 20:19-23.

8. Shin $\mathrm{SH}$, Lee $\mathrm{YH}$, Jeon $\mathrm{CH}$. Proteasedependent activation of nasal polyp epithelial cells by airborne fungi leads to migration of eosinophils and neutrophils. Acta Oto-Laryngol. 2006; 126:1286-1294.

9. Daines MO, Flynn A, Sherwood C, et al. PAR-2 activation by alternaria alternata proteases induces airway epithelial cell activation and lung inflammation. Conference: 2011 American Academy of Allergy, Asthma and Immunology, AAAAI Annual Meeting San Francisco, CA United States. J. Allergy Clin. Immunol. 2011; 127(S2):AB59.
Mr Rami J. Salib

Associate Professor in Rhinology \&

Consultant ENT Surgeon

Academic Unit of Clinical \& Experimental Sciences

Department of Otorhinolaryngology Head \& Neck Surgery

University Hospital Southampton NHS Foundation Trust

Tremona Road

Southampton

SO16 6YD, United Kingdom

Tel: $+44-23-80825635$

Fax: +44-23-8082 5688

E-mail: R.J.Salib@soton.ac.uk 